\title{
Optimal Measurement Strategies for Effective Suppression of Drift Errors
}

\author{
Valeriy V. Yashchuk \\ Lawrence Berkeley National Laboratory, Berkeley, CA 94720*
}

(Dated: April 16, 2009)

\begin{abstract}
Drifting of experimental set-ups with change of temperature or other environmental conditions is the limiting factor of many, if not all, precision measurements. The measurement error due to a drift is, in some sense, in-between random noise and systematic error. In the general case, the error contribution of a drift cannot be averaged out using a number of measurements identically carried out over a reasonable time. In contrast to systematic errors, drifts are usually not stable enough for a precise calibration. Here a rather general method for effective suppression of the spurious effects caused by slow drifts in a large variety of instruments and experimental set-ups is described. An analytical derivation of an identity, describing the optimal measurement strategies suitable for suppressing the contribution of a slow drift described with a certain order polynomial function, is presented. A recursion rule as well as a general mathematical proof of the identity is given. The effectiveness of the discussed method is illustrated with an application of the derived optimal scanning strategies to precise surface slope measurements with a surface profiler.
\end{abstract}

PACS numbers: 06.30-k, 42.72.-g, 42.87.-d 


\section{INTRODUCTION}

Consider a measurement of the functional dependence $Q(v)$ of a physical quantity $Q$ on a variable $v$ that is performed by scanning the variable over discrete points $v_{i}, i=0,1, \ldots,(I-$ 1), uniformly distributed over the range of interest (ROI) of the function. Generally, the measured quantities $Q_{M}\left(v_{i}\right)$ are affected by random errors, $R\left(v_{i}\right)$, systematic error, $S\left(v_{i}\right)$, and drift, $D\left(v_{i}\right)$, of the measurement instrument and experimental set-up:

$$
Q_{M}\left(v_{i}\right)=Q\left(v_{i}\right)+R\left(v_{i}\right)+S\left(Q\left(v_{i}\right), v_{v i}\right)+D\left(v_{i}(t)\right) .
$$

Random errors are caused by unpredictable fluctuations in the readings of the measurement instrument due to a limited precision of the instrument and/or due to the random character of the measurement quantity. ${ }^{1,2}$ If the random fluctuations are fast compared to the time available for the measurement, the contribution of random errors can, in principle, be made as small as required simply by averaging multiple sequential scans $Q_{M, s}\left(v_{i}\right)$ carried out at the same experimental conditions:

$$
\widehat{Q}_{M}\left(v_{i}\right)=\frac{1}{S} \sum_{s=1}^{S} Q_{M, s}\left(v_{i}\right),
$$

where symbol $s$ denotes the number of the scan, $s=1,2, \ldots, S$, and $S$ is the total number of scans of the measurement run.

The systematic error in Eq. (1) is a part of the measurement error that is systematically reproduced in the sequential scans and, therefore, cannot be suppressed by averaging over repeatable measurements given by Eq. (2). Systematic errors are usually associated with insufficient calibration of the instrument for a specific experimental arrangement. Sometimes, reliable calibration of the instrument is rather sophisticated, for example, calibration of surface slope measuring profilers discussed in Ref. ${ }^{3}$ In any case, precision testing and calibration of the experimental set-up is, practically, the only reliable way to illuminate (account for) the systematic errors.

Instrumental and set-up drifts are the limiting factors of many, if not all, precise measurements requiring collection of data for extended period of time. The drift errors are caused by a relatively slow variation of the experimental conditions such as temperature, humidity, etc. The error due to a drift is, in some sense, in-between the random noise and systematic error. Unlike a random noise, the error contribution of a drift cannot be averaged out using 
multiple scans identically carried out over a reasonable time. In contrast with systematic errors, drifts are usually not stable enough for accounting via a precise calibration.

In the present article, we describe an experimental method for effective suppression of the spurious effects caused by slow drifts. One of the realizations of the method, considered in detail in Sec. II, utilizes an idea to perform repeatable measurements at a certain point $v_{i}$ at a sequence of time moments $t_{i}(s)$, specially arranged to anti-correlate with the temporal dependence of the drift. The required sequence of the time intervals over which measurements are performed is arranged with sequential reversals of the direction of scanning towards increase or decrease of $v_{i}$. An identity that describes a sequence of reversed scans, suitable for suppressing a contribution of a slow drift described with a polynomial function of a certain order, is analytically derived in Sec. II. In Sec. III, a recursion rule, as well as a general mathematical solution (see also Appendix) of the identity are given. The effectiveness of the discussed method is illustrated in Sec. IV by the application of the derived optimal scanning strategies to precise surface slope measurements affected by a slow exponential drift. In Sec. V, we discuss suppression of the drift error in an experiment, where the measured physical quantity itself can be reversed. That means reversing the sign of the recorded quantity without changing the sign of the drift error. We show that the optimal sequences of the sign reversals also obey the same identity as the one derived in Sec. II for reversing the direction of scanning. Note that the derived optimal sequences of the reversals also describe the optimal square waveforms for drift-free multichannel phase-sensitive detection discussed in Ref. ${ }^{4}$ and first used in Refs. ${ }^{5,6}$ in an experiment searching for parity (P) and time reversal invariance $(\mathrm{T})$ violating electric dipole moment of xenon. Since then, this technique has been widely used in experiments searching for $\mathrm{P}$ and $\mathrm{T}$ violating electric dipole moments in neutrons, ${ }^{7,8}$ atoms,${ }^{9-11}$, molecules, ${ }^{12,13}$ and solids. ${ }^{14}$ In Sec.V, we show that simultaneous optimal reversal of the direction of scanning and orientation of a surface under test (SUT) provides drift-free surface metrology with slope-measuring profilers. A profiler that would allow such reversals is under construction at the Advanced Light Source (ALS) Optical Metrology Laboratory (OML). In Sec. VI, the effectiveness of the derived optimal scanning strategies for a real experimental arrangement is illustrated with the ALS DLTP (Developmental Long Trace Profiler ${ }^{15}$ ) using measurements with a high quality reference mirror. A comparison with the corresponding results obtained with the world's best slope measuring instrument, the BESSY NOM,${ }^{16}$ proves the accuracy of the DLTP measurements 
to be about $0.1 \mu \mathrm{rad}$ (peak-to-valley) in spite of the presence of 25 - $\mu \mathrm{rad}$ set-up drifts during a measurement.

\section{DRIFT ERROR SUPPRESSION BY REVERSING SCANNING DIRECTION}

\section{A. Slow drifts}

We assume that a slow drift is described with a function $D(t)$ that can be presented as a MacLaurin polynomial series:

$$
D(t)=\sum_{n=0} \frac{D^{(n)}(0)}{n !} t^{n}=\sum_{n=0} d_{n} t^{n} .
$$

The possibility for a drift to be represented as a MacLaurin series (existence of derivatives up to rather high orders) seems to be very natural. Moreover, a large class of drifts can be described with an exponential function that has derivatives which exist for any order:

$$
D(t)=D_{0} \exp (-t / \tau)=D_{0} \sum_{n=0}(-1)^{n} \frac{t^{n}}{\tau^{n} n !}=\sum_{n=0} d_{n} t^{n},
$$

where $\tau$ is a time constant. Throughout the present work, an exponential drift is slow, if a duration of a scan, $\Delta T$, is smaller than $\tau$ :

$$
\Delta T=I \delta t<\tau
$$

where $\delta t$ is an incremental time between the sequential measurements and $I$ is the total number of measurements per scan.

If a drift is fast, i.e. $I \delta t \geq \tau$, the best strategy is to wait for a few periods of $\tau$, when the experimental conditions become stable enough for the required accuracy of measurements. Practically, such a strategy has been realized in the DLTP data acquisition system by introducing a delay time before starting a run. ${ }^{15}$ Suppression of a slow exponential drift is analyzed in Sec. IV in more detail.

For a general case of a slow drift described with a MacLaurin series (3), a condition analogous to (5) can be written as a limit of the derivatives:

$$
D^{(n)}(0)(\Delta T)^{n}<1
$$

Below, we describe an experimental method for minimizing a certain order of polynomial drift. The idea of the method is to average repeatable measurements of a physical quantity 
at a certain point $v_{i}$ performed at a sequence of time moments $t_{i}(s)$, specially arranged to anti-correlate with the temporal dependence of the drift. The results of this section are applicable in a broad class of measurements, including (but not limited to), for example, mapping electric-potential distributions with a Laplace's bath, ${ }^{17,18}$ high-precision metrology of x-ray optics with long trace profilers, ${ }^{19,20}$ etc.

As a concrete example, we consider a measurement of surface topography with a slopemeasuring profiler. Figure 1 shows a simplified schematic of the experimental set-up. The optical slope sensor of the profiler is a high precision autocollimator, which is stationary on an optical table. Scanning along a surface under test (SUT) is performed by translating a pentaprism mounted on a translation stage. The translation stage and the motion-control system of the profiler allow for scanning in the forward and in the backward directions. The schematic in Fig. 1 corresponds to, for example, to the NOM (Nanometer Optical Component Measuring machine) slope profiler at BESSY (Germany), ${ }^{16}$ the ESAD (Extended Shear Angle Difference) profiler at the PTB (Germany), ${ }^{21}$ and the DLTP recently developed at the ALS OML. ${ }^{15}$

\section{B. Optimal scanning sequences}

Consider a slope profile measured in a single $s$-th scan of the profiler (Fig. 1), $\alpha_{s}\left(x_{i}\right)$ with a total number of measured points of $I$, and $i=\{0, . ., I-1\}$. The resulting trace is obtained by averaging over S scans, $s=\{1, . ., S\}$, of the run:

$$
\hat{\alpha}_{i}=\frac{1}{S} \sum_{s=1}^{S} \alpha_{s}\left(x_{i}\right),
$$

Analogously to Eqs.(1) and (2), the resulting slope trace $\hat{\alpha}_{i}$ can be thought of as a sum of a trace of the SUT surface slopes, $\alpha_{i}^{0}$, and contributions of random noise, $\alpha_{i}^{R}$, systematic errors, $\alpha_{i}^{S}$, of the measurement, and drifts, $\alpha_{i}^{D}$, of the set-up:

$$
\hat{\alpha}_{i}=\alpha_{i}^{0}+\alpha_{i}^{R}+\alpha_{i}^{S}+\alpha_{i}^{D}
$$

Precise measurement of $\alpha_{i}^{0}$ assumes insignificant contributions of the random noise and systematic errors, and we don't consider them, concentrating on minimization of the error related to drifts. 
Assuming that the measured points are uniformly distributed along the SUT, the set of time moments, when the $i$-th point is measured during the $s$-th scan performed in the forward direction, can be described as

$$
t_{i}^{F}(s)=\delta t[(s-1) \cdot I+i]
$$

If the $s$-th scan is performed in the backward direction, equation (8) transforms to

$$
t_{i}^{B}(s)=\delta t[s \cdot I-i]
$$

We describe a sequence of directions of scans in a measurement run with a binary sequence $\left\{r_{s}\right\}$ with the elements:

$$
r_{s}= \begin{cases}+1 & \text { if the } s \text {-th scan is performed in the forward direction; } \\ -1 & \text { if the } s \text {-th scan is performed in the backward direction }\end{cases}
$$

We will say that the binary sequence $\left\{r_{s}\right\}$ describes a scanning strategy for the corresponding run.

Using the notation (11), the equations (9) and (10) can be rewritten in more generalized form:

$$
t_{i}(s)=\delta t\left[\left(s-\frac{1+r_{s}}{2}\right) \cdot I+r_{s} i\right]=\delta t\left[(s-0.5) \cdot I+r_{s}(i-0.5 I)\right] .
$$

The factor $\delta t$ in (12) is a time scale factor that we assume to be $\delta t=1$; and, therefore, it can be omitted in (12) without loss of generality:

$$
t_{i}(s)=(s-0.5) \cdot I+r_{s}(i-0.5 I)
$$

Consider a general case of the $n$-th order drift described with (3). After averaging over all $S$ scans of the run, a contribution of the drift to a slope value measured in the position point $x_{i}$ is

$$
D_{n}\left(x_{i}\right)=\frac{d_{n}}{S} \sum_{s=1}^{S} t_{s}^{n}(s)=\frac{d_{n}}{S} \sum_{s=1}^{S}\left[(s-0.5) \cdot I+r_{s}(i-0.5 I)\right]^{n} .
$$

After simple algebraic transformations, (14) can be rewritten as

$$
\begin{aligned}
D_{n}\left(x_{i}\right) & =\frac{d_{n}}{S} \sum_{k=0, \text { even }}^{n} I^{n-k} C_{n}^{k}(i-0.5 I)^{k} \sum_{s=1}^{S}(s-0.5)^{n-k} \\
& +\frac{d_{n}}{S} \sum_{k=1, \text { odd }}^{n} I^{n-k} C_{n}^{k}(i-0.5 I)^{k} \sum_{s=1}^{S} r_{s}(s-0.5)^{n-k},
\end{aligned}
$$


where $C_{n}^{k} \equiv\left(\begin{array}{l}n \\ k\end{array}\right) \equiv \frac{n !}{(n-k) ! k !}, 0 \leq k \leq n$, are the binomial coefficients. In order to zero out the second term in (15), the scanning sequence $\left\{r_{s}\right\}$ should obey the identity below with a certain value of total number of scans, $S$,

$$
\sum_{s=1}^{S} r_{s}(s-0.5)^{n-k} \equiv 0 \quad \text { for } \quad 1 \leq k \leq n
$$

In the next section a recursion rule for finding the optimal scanning sequences is presented. A stronger proof for the existence of the two solutions $\left\{r_{s}^{+}\right\}$and $\left\{r_{s}^{-}\right\},\left\{r_{s}^{+}\right\}=-\left\{r_{s}^{-}\right\}$, for the identity (16) and analytical forms of the solutions are derived in the Appendix. It is shown that if $\left\{r_{s}\right\}$ is a solution of the identity

$$
\sum_{s=1}^{S} r_{s} s^{n-1} \equiv 0, \quad \text { for any natural } n
$$

it is also a solution of the identity

$$
\sum_{s=1}^{S} r_{s}(s+p)^{n-k} \equiv 0
$$

with $S=2^{n}$, for natural $k \leq n$ and any real $p$.

Therefore, from (18) at $p=0.5$ and $S=2^{n}$, the second term of (15) can always be zeroed out with an appropriate choice of scanning strategy $\left\{r_{s}\right\}$.

The first term of (15) is not affected by the scanning procedure and should be analyzed as a residual error due to the drift. This term can be presented as a sum of an offset term with $k=0$, independent of position on the mirror surface, and the rest of the sum that describes the residual drift error, varying from point to point:

$$
D_{n}\left(x_{i}\right)=\frac{d_{n}}{S} I^{n} \sum_{s=1}^{S}(s-0.5)^{n}+\frac{d_{n}}{S} \sum_{k=2, \text { even }}^{n} I^{n-k} C_{n}^{k}(i-0.5 I)^{k} \sum_{s=1}^{S}(s-0.5)^{n-k}
$$

The offset term only gives an overall offset of the measured slope trace. The offset does not perturb the surface slope metrology and can be thought of as an overall tilt of the SUT.

The highest order (on $S$ ) of the drift error $(19)$ is $(n-2)$. This can be compared with the systematic error for the worst case of a totally non-optimized run of the same number of scans but performed without reversing the scan direction that that has order of $(n-1)$ on $S$. This is obtained from (15) by using $r_{s} \equiv 1$ for all $s$. The corresponding suppression factor can be expressed as a ratio of peak-to-valley variations $(P V V)$ of the major terms of 
the drift error of the optimized and non-optimized runs of the same total number of scans:

$$
\xi \approx \frac{P V V\left[I^{n-1} C_{n}^{1}(i-0.5 I) \sum_{s=1}^{S} r_{s}(s-0.5)^{n-1}\right]}{P V V\left[I^{n-2} C_{n}^{2}(i-0.5 I)^{2} \sum_{s=1}^{S}(s-0.5)^{n-2}\right]} \propto 8 \frac{2^{n}}{n} .
$$

The estimation (20) shows that suppression factor, $\xi$, rapidly increases with increase of $n$ for $n \geq 2$. Below, we apply the result of the general consideration above to the particular cases of polynomial drifts of the first few orders.

\section{Delay time between sequential scans}

So far we have assumed that the delay time between sequential scans due to reversing is negligible compared to all other time scales in the problem. In this section, the case of non-zero delay time between the scans is analyzed.

An arbitrary pattern of delay time between scans can be analyzed with an additional term in expression (13) for the set of time moments of measurements in the $i$-th spatial point:

$$
t_{i}(s)=(s-0.5) \cdot I+r_{s}(i-0.5 I)+a_{s} \varepsilon I,
$$

where $\left\{a_{s}\right\}$ is a sequence of real numbers describing a total delay for the $s$-th scan, and the delays are expressed as a fraction $\varepsilon$ of the duration time $\Delta T$ of a single scan:

$$
\varepsilon I \delta t=\frac{\Delta I}{I} I \delta t
$$

First, consider a constant delay before each scan (a delay before the first scan does not change anything and we apply it to simplify the following expressions). This can be accounted in (21) with a delay sequence:

$$
\left\{a_{s}\right\}=\{s\}
$$

Then, relation (21) can be presented as

$$
t_{i}(s)=\left(s-\frac{0.5}{1+\varepsilon}\right) \cdot I(1+\varepsilon)+r_{s}(i-0.5 I)
$$

which is basically identical to (13). Therefore, the conditions for optimal scanning strategy

are still given by (18) with $p=-\frac{0.5}{1+\varepsilon}$ and the optimization identities and their solutions $\left\{r_{s}\right\}$ are the same as discussed in Sec. II. 
If the delay time between sequential scans is significantly smaller than the duration of a single scan $(\varepsilon<<1)$, one can neglect $\varepsilon$ in $(24)$ and use the results of Secs. II- IV for estimation of the suppression efficiency.

For completeness, consider a case of measurements when a significant delay $(\varepsilon \approx 1)$ appears only between two sequential scans in the same direction. This situation corresponds to surface profiling with a significant time delay for returning the carriage to the previous start position. Then, the delay sequence can be expressed as:

$$
a_{s}=\sum_{j=1}^{s}\left(\frac{r_{j-1}^{+}+r_{j}^{+}}{2}\right)^{2}=\sum_{j=1}^{s}\left(1+r_{j-1}^{+} \cdot r_{j}^{+}\right),
$$

assuming $r_{j=0}=-1$. The first few elements of the sequence $\left\{a_{s}\right\}$ are

$$
\left\{a_{s}\right\}=\{0,0,2,2,2,2,4,4,6,6,8,8,8,8,10,10, \ldots\}
$$

In this case, in the expression, analogous to (15), there would be additional terms that are zeroed with the found optimal scanning patterns due to the identity:

$$
\sum_{s=1}^{2^{n}} r_{s}\left(a_{s}\right)^{n-1} \equiv 0
$$

However, there would also be more additional terms that contribute to the residual drift error. Analysis of the contributions is rather cumbersome and we omit it here.

\section{RECURSION RULE FOR FINDING OPTIMAL SCANNING STRATEGIES}

\section{A. Suppression of a linear drift}

Let us first consider a linear drift described in (3) with $d_{1} t$. After averaging over all $S$ scans of the run, the contribution of linear drift to a slope value measured for a position point $x_{i}$ is

$$
D_{1}\left(x_{i}\right)=\frac{d_{1}}{S} \sum_{s=1}^{S} t_{i}(s)=\frac{d_{1}}{S}\left[I \sum_{s=1}^{S}(s-0.5)+(i-0.5 I) \sum_{s=1}^{S} r_{s}\right] .
$$

The first term in (28) does not depend on the point index $i$; and, therefore, it does not perturb the surface slope metrology of the SUT.

The second term in (28) can be easily zeroed if

$$
\sum_{s=1}^{S} r_{s} \equiv 0
$$


This is a trivial result that in order to suppress a linear drift, a scanning strategy $\left\{r_{i}\right\}$ should include the same number of scans in the forward and in the backward directions; the succession of the scans is not important. The simplest realizations of the strategy are two-scan runs:

$$
\left\{r_{s}^{+}\right\}=\{+1,-1\} \equiv\{F, B\} \text { and }\left\{r_{s}^{-}\right\}=\{-1,+1\} \equiv\{B, F\}
$$

\section{B. Suppression of a quadratic drift}

Second, consider a quadratic drift described in (3) with $d_{2} t^{2}$ :

$$
\begin{aligned}
& D_{2}\left(x_{i}\right)=\frac{d_{2}}{S} \sum_{s=1}^{S} t_{i}^{2}(s)=\frac{d_{2}}{S} I^{2} \sum_{s=1}^{S}(s-0.5)^{2}-\frac{d_{2}}{S} I(i-0.5 I) \sum_{s=1}^{S} r_{s} \\
& +\frac{d_{2}}{S} 2 I(i-0.5 I) \sum_{s=1}^{S} r_{s} s+d_{2}(i-0.5 I)^{2} .
\end{aligned}
$$

The first term in (31) does not depend on the point index and, therefore, gives an overall offset of the measured slope trace.

The second term in (31) is zeroed, if the scanning strategy $\left\{r_{i}\right\}$ includes the same number of scans in the forward and in the backward directions. This requirement is the same as condition (30) for an optimal strategy suppressing a linear drift.

The third term in (31) can also be zeroed, if the scanning strategy $\left\{r_{i}\right\}$ obeys the additional condition

$$
\sum_{s=1}^{S} r_{s} s \equiv 0 .
$$

It is easy to directly check that the simplest solutions of Eq. (32) that also obey (29) are the strategies of four-scan runs:

$$
\begin{aligned}
& \left\{r_{s}^{+}\right\}=\{+1,-1,-1,+1\} \equiv\{F, B, B, F\} \text { and } \\
& \left\{r_{s}^{-}\right\}=\{-1,+1,+1,-1\} \equiv\{B, F, F, B\} .
\end{aligned}
$$

The fourth term in (31) is a residual drift error due to the quadratic drift. The error does not depend on the total number of scans. It should be compared with the result obtained by averaging over 4 scans of a non-optimized run, $\left\{r_{i}\right\} \equiv 1$, carried out without reversing the scanning direction:

$$
D_{2}^{\times}\left(x_{i}\right)=d_{2}(i-0.5 I)^{2}+d_{2} S I(i-0.5 I),
$$

that has an additional term linearly increasing with the increase of the total number of scans in the run. The cross index in (34) denotes that the error was estimated for a non-optimized 
run. Numerical comparison of the last term in (31) with the drift error (34) suggests that a minimal optimum run of four scans (33) allows suppression of the peak-to-valley variation due to the quadratic drift by a factor of about 18 .

\section{Suppression of a third-order drift}

In the same straightforward way that was used for the first- and the second-order drifts, we can consider a third-order drift described in (3) with term $d_{3} t^{3}$. After averaging over all $S$ scans of the run, the contribution of the third-order drift to a slope value measured for the position point $x_{i}$ is

$$
\begin{aligned}
D_{3}\left(x_{i}\right)= & \frac{d_{3}}{S} \sum_{s=1}^{S} t_{i}^{3}(s) \\
= & \frac{d_{3}}{S} I^{3} \sum_{s=1}^{S}(s-0.5)^{3}+\frac{d_{3}}{S}(i-0.5 I)\left[i^{2}-i I+I^{2}\right] \sum_{s=1}^{S} r_{s} \\
& -\frac{d_{3}}{S} 3 I^{2}(i-0.5 I) \sum_{s=1}^{S} r_{s} s+\frac{d_{3}}{S} 3 I^{2}(i-0.5 I) \sum_{s=1}^{S} r_{s} s^{2} \\
& +d_{3} \frac{3}{2} I(i-0.5 I)^{2} S
\end{aligned}
$$

The first three terms in (35) are similar to that have obtained in (31) for a quadratic drift. These terms does not perturb slope measurements if the conditions (29) and (32) are fulfilled for the used scanning strategy.

The fourth term in (35) is zeroed, if the scanning strategy $\left\{r_{i}\right\}$ additionally obeys a new condition

$$
\sum_{s=1}^{S} r_{s} s^{2} \equiv 0 .
$$

One can directly check that the simplest solutions of Eq. (36) that also obey (29) and (32) are the strategies of eight-scan runs:

$$
\begin{aligned}
& \left\{r_{s}^{+}\right\}=\{+1,-1,-1,+1,-1,+1,+1,-1\} \equiv\{F, B, B, F, B, F, F, B\} \text { and } \\
& \left\{r_{s}^{-}\right\}=\{-1,+1,+1,-1,+1,-1,-1,+1\} \equiv\{B, F, F, B, F, B, B, F\} .
\end{aligned}
$$

The fifth term in (35) is a residual drift error due to the quadratic drift. The error does depend on the total number of scans. However, the magnitude of the error is significantly, by factor of about 21 [see also Eq. (20)], suppressed compared with the drift error that would be obtained by averaging over the same number of scans of a non-optimized run, carried out without reversing the scanning direction:

$$
D_{2}^{\times}\left(x_{i}\right)=d_{3}(i-0.5 I) i(i-I)-\frac{3}{2} d_{3} I(i-0.5 I)(2 I-i) S+d_{3}(i-0.5 I) I^{2} S^{2} .
$$


The non-optimized error (38) has an additional term quadratically increasing with the increase of the total number of scans in the run. This term would give a major spurious contribution from the third order drift to the slope measurement. This contribution is effectively eliminated with the scanning strategies depicted with the binary sequences (37).

\section{Recursion Rule}

Note first that the optimal strategies $\left\{r_{s}^{+}\right\}$and $\left\{r_{s}^{-}\right\}$given with (30), (33), and (37) obey the identity

$$
\left\{r_{s}^{-}\right\}=(-1)\left\{r_{s}^{+}\right\}
$$

There is a simple recursion rule for the found optimal strategies that was directly verified from the above consideration for the first three orders. An optimal strategy $\left\{r_{s}^{+}(n+1)\right\}$ for suppression of a polynomial drift of $(n+1)$-th order is obtained by stitching together the solutions $\left\{r_{s}^{+}(n)\right\}$ and $\left\{r_{s}^{-}(n)\right\}$ found for the previous order:

$$
\left\{r_{s}^{+}(n+1)\right\}=\left\{r_{s}^{+}(n), r_{s}^{-}(n)\right\}
$$

Together with (39), the recursion rule (40) allows construction of the optimal scanning strategies to suppress a slow drift up to any desired order of the MacLaurin series of the temporal dependence of the drift.

\section{SUPPRESSION OF SLOW EXPONENTIAL DRIFT}

The efficiency of the found optimal strategies can be numerically demonstrated assuming a slow exponential drift described with

$$
D(t)=D_{0} \exp (-t / \tau)
$$

avoiding any limitation related to an approximation with a limited MacLaurin series.

Figure 2 illustrates the exponential drift suppression when a measurement run consists of only four sequential scans depicted with the strategy $\left\{r_{s}^{+}\right\}=\{+1,-1,-1,+1\} \equiv$ $\{F, B, B, F\}$ given by (33) and optimal for suppression of the second order polynomial drift.

In Fig. 2a, exponential drift described by Eq. (41) with the parameters $D_{0}=0.5 \mu \mathrm{rad}$ and $\tau=60 \mathrm{~min}$ is shown. The simulation assumes that during two hours, four sequential 
measurements are performed according to the scanning strategy $\left\{r_{s}^{+}\right\}=\{F, B, B, F\}$ to measure a slope distribution along a SUT with the length of $300 \mathrm{~mm}$. Therefore, duration of a scan of 30 min fulfills the condition (5) for a slow exponential drift.

Because the directions of the second and the third scans are reversed, the corresponding contributions of the drift to the resulting slope trace are also reserved. That is depicted in Fig. 2b. The resulting trace of the drift slope error due to the exponential drift measured in the optimized four-scan run is shown with the red line and points in Fig. 2c. For comparison, the blue line and points in Fig. 1c present the drift slope trace that would be measured in a non-optimized run of four repeatable scans performed in the same direction.

The efficiency of drift suppression in the considered example is $\xi \approx 7.5$. Averaging with an additional run performed with the scanning strategy $\left\{r_{s}^{-}\right\}=\{B, F, F, B\}$ would correspond to a run with the strategy $\left\{r_{s}^{+}\right\}=\{F, B, B, F, B, F, F, B\}$, optimized for suppression of polynomial drifts up to third order [compare with expression (37)]. In the case of the exponential drift, simulated here, the overall suppression factor after 8 optimal scans would be $\xi \approx 8.7$. The improvement is not so dramatic because of the small amplitude of the third order polynomial in the MacLaurin series of a slow exponential drift.

\section{DRIFT ERROR SUPPRESSION BY REVERSING MEASURING QUANTITY}

In the experiment shown in Fig. 1, the direction of a scan can be changed by reversing the scanning direction of the profiler or by changing the orientation of the SUT. From the point of view of optimization of the scanning strategy of the slope measurement in Fig. 1, these two options are not absolutely identical. If the scanning direction of a profiler is reversed, the contribution to the measured slope value due to the inherent mirror surface profile, $\alpha_{i}^{0}$ in (8), remains unchanged. This is in contrast to the change of the SUT orientation, when the slope contribution due to the SUT changes in its sign. Optimal scanning strategies for the case of reversing the scanning direction of a profiler were analyzed in Sec. II. The advantages, possible with the additional reversal of changing the SUT orientation, are discussed below.

First, let us consider reversing the scanning direction performed by only changing orientation of the SUT. In this case, the contribution of the $n$-th order polynomial drift to the 
slope value measured in the position point $x_{i}$ is [compare with Eq. (14)]

$$
D_{n}\left(x_{i}\right)=\frac{d_{n}}{S} \sum_{s=1}^{S} r_{s}\left[(s-0.5) \cdot I+r_{s}(i-0.5 I)\right]^{n},
$$

where the additional term $r_{s}$ accounts for the sign change in the course of averaging over the scans of the run. It is straightforward to show with a consideration, similar to one presented in Sec. II that the orientation reversal leads to suppression of the first term in (15) rather than the second term of larger order on $S$. Therefore, the overall suppression would be smaller than one obtained by reversing the scanning direction of the profiler.

However, a strategy that includes both reversals can allow absolute zeroing of the driftrelated errors.

For example, if in the course of a slope measurement the scanning direction and the orientation of the SUT are reversed with the same strategy $\left\{r_{s}(n)\right\}$, the drift error contribution is

$$
D_{n}\left(x_{i}\right)=\frac{d_{n}}{S} \sum_{s=1}^{S} r_{s}[(s-1) \cdot I+i]^{n} .
$$

Similar to Eq. (42), the term $r_{s}$ in Eq. (43) accounts for the change of the SUT slope sign when the orientation of the SUT is reversed. Because the scanning direction of the profiler is simultaneously changed with the same strategy, the scanning direction with respect to the SUT surface is not changed; and the time sequence of the run should correspond to scanning in the same direction. In Eq. (43), this is the forward direction scanning described with (9). The equation (43) is equivalent to

$$
D_{n}\left(x_{i}\right)=\frac{d_{n}}{S} \sum_{k=0}^{n} I^{n-k} C_{n}^{k} i^{k} \sum_{s=1}^{S} r_{s}(s-1)^{n-k},
$$

that, as it was shown in Sec. II and more strongly proven in the Appendix, can be identically zeroed using the optimal scanning strategies $\left\{r_{s}(n)\right\}$ derived and discussed throughout the present article [compare with Eq. (18)].

In order to realize such a scanning strategy with the LTP or DLTP slope measuring instruments, a corresponding upgrade should be made to allow automatic and simultaneous change of the orientation of a surface under test and the scanning direction of the profiler. A profiler that would allow such reversals is under construction at the ALS OML. Note that reversing the SUT orientation can provided a suppression of the systematic error. ${ }^{22}$ 
We can now formulate that the most efficient suppression of drift error (in fact, identical zeroing of polynomial drifts up to a desired order) is obtained with sequential scans with optimal reversing of the sign of the measuring quantity without changing the sign of the drift error contribution. The optimal sequences of the sign reversals also obey the same identity as the one derived in Sec. II for reversing the direction of scanning.

\section{DLTP MEASUREMENTS WITH REVERSING SCANNING DIRECTION}

In order to illustrate the effectiveness of the above optimal scanning strategies for a real experimental arrangement, we describe below DLTP measurements (Fig.1) with the

S3 reference mirror, ${ }^{23-25}$ and provide a comparison with the corresponding results obtained with the world's best slope measuring instrument, the BESSY NOM. ${ }^{16}$

The DLTP experiment with the S3 reference mirror consisted of a total of six overnight measurement series, each composed of multiple scans. A few precautions were used in order to decrease the possible systematic errors. ${ }^{15}$ First, three measurement series were carried out with the direct orientation of the mirror, and three measurement series with the reversed mirror orientation. Second, for each measurement series of the total six, the S3 mirror was re-aligned to have a different tangential tilt that appears in a corresponding slope trace as an offset. It should be mentioned that because of the drift of the profiler set-up, the tilt offset is changing from scan to scan composing a measurement. Ironically, we believe this circumstance to be helpful for better averaging of the DLTP systematic errors. Each measurement series consists of 16 scans total, including four consequent series of F-B-BF runs. The measurements were usually started at $6 \mathrm{PM}$ and finished by approximately $10 \mathrm{AM}$ of the next day in order to carry out an overnight measurement at significantly quieter industrial noise. (There is constraction of a new building next door.)

The preliminary tests of the $\operatorname{DLTP}^{15}$ have shown a significant dependence of the performance of the instrument on the laboratory environmental conditions, that is mainly a slow drift of the room temperature with a diurnal peak-to-value variation up to a few degrees Centigrade. Figure 3 illustrates the repeatability of measurements inside a measurement series via a difference of the slope traces for the first (1st) and the last (16th) scans of the same measurement series. There is a significant change of offset that is about $25 \mu \mathrm{rad}$ for the tangential slope traces measured with an interval of approximately 15 hours. This big 
change is caused by a temperature drift of the mutual alignment of the mirror and the autocollimator due to temperature instability of the DLTP optical table upon the diurnal temperature drift in the lab.

At first glance, keeping in mind the requirement for sub-microradian accuracy of DLTP slope measurements, we have a deadlocked situation. Nevertheless, an excellent match of the averaged measurements performed with the ALS DLTP and BESSY NOM ${ }^{26}$ is clearly seen in Fig. 4, which is the result of the drift suppression by the optimal measurement strategy. Moreover some deviations of the measurements can be attributed to the difference in the increment of the slope profiles. BESSY data are shown with an increment of $1 \mathrm{~mm}$, while the ALS data are plotted with increment of $0.2 \mathrm{~mm}$. By and large, the difference of the measurements does not exceed $0.1 \mu \mathrm{rad}$ (peak-to-valley).

We specially compare the ALS DLTP measurements with BESSY NOM results because the known high performance of the NOM is guaranteed by the extremely sophisticated design of the profiler and extremely quiet environmental conditions inside the BESSY optical metrology laboratory, and specially around the instrument. ${ }^{16}$ These became possible due to the massive investment in development of the NOM, unprecedented careful design of every single part of the NOM set-up, as well as due to the profiler calibration additional to the calibration of the NOM autocollimator. In the measurement with the ALS DLTP (that is of significantly less cost), a comparable accuracy became possible by implementing a sophisticated measurement strategy, discussed throughout the present work.

\section{DISCUSSION AND CONCLUSIONS}

We have considered a rather general method for effective suppression of the spurious effects caused by slow drifts in a large variety of experiments. The method is based on the application of scanning strategies capable of suppression of polynomial drifts up to a certain desired order. A general form of identities describing a set of optimal scanning strategies has been derived. A simple recursion rule for finding the solutions of the identities has been also suggested. It has been also shown that the same optimal scanning strategies allow identical zeroing of polynomial drifts up to the desired order if reversing the sign of the measuring quantity without changing the sign of the drift error contribution is used.

The high effectiveness of the found optimal scanning strategies has been numerically 
verified for the case of low order polynomial drifts, as well as for the case of a slow exponential drift. The performed consideration is limited with a case of slow drifts. That guarantees that the contribution of a certain order polynomial to the drift error relatively decreases with increase of the order. Basically, this allows application of the described approach to any slow drift with a temporal dependence that can be approximated with a limited MacLaurin series of polynomials.

The advantages of use of the discussed optimal scanning strategies in a real experiment was illustrated with the DLTP surface slope metrology of a super high quality reference mirror. A comparison with the corresponding results obtained with the world's best slope measuring instrument, the BESSY NOM, proves the accuracy of the DLTP measurements to be about $0.1 \mu \mathrm{rad}$ (peak-to-valley) in spite of the presence of $25 \mu \mathrm{rad}$ set-up drifts during a measurement. The demonstrated performance of the DLTP is mostly due to the original measurement strategy, as it has been discussed throughout the present work, that is capable for effective suppression of the set-up drifts and systematic errors. However, it does not follow from our results that the DLTP is a similar quality instrument to NOM, at least, not for entire angular range. The rather large radius of curvature (about $1280 \mathrm{~m}$ ) of the S3 reference mirror does not allow testing the total slope range of the DLTP (about $\pm 5 \mathrm{mrad}$ ) where one should expect the systematic errors to be significantly larger. Moreover, if the same strategy and finesses are applied while measuring with the NOM, the result is expected to be also significantly improved.

In conclusion, we have discussed an experimental method for suppressing drift-related errors. We believe that a similar suppression of the drift errors can be also obtained via a sophisticated numerical treatment of a set of the sequential scans. In this case, one could use, for example, the method of maximum likelihood to find a best fitted polynomial approximation of the error contribution. A discussion of this approach is, however, beyond the scope of the present work.

\section{APPENDIX A: GENERAL PROOF FOR THE CORRELATION IDENTITY 27}

The correlation identity to be proven can be written in a simple from:

$$
\sum_{s=1}^{2^{n+1}} r_{s} s^{n} \equiv 0
$$


where $s$ and $n$ are natural numbers, $\left\{r_{s}\right\}$ is a binary sequence of +1 s and -1 s of the total length of $2^{n+1}$. The physical meaning of the parameters in (A1) is following. Index $s$ is the index number for the $s$-th scan. The binary array $\left\{r_{s}\right\}$ describes a sequence of directions of scanning (reversals) - Eq.(11). Index $n$ is the order of a polynomial term of the MacLaurin series of the drift function to be suppressed with the scanning pattern given with $\left\{r_{s}\right\}$. A measurement run consists of the total number of scans of $S=2^{n+1}$ that have to be averaged to get the resulting measurements, maximally free of an error due to the drift.

The solution presented below is based on the well known property of finite difference derivatives of a polynomial that the finite-difference partial derivative of a polynomial of degree $k$ is a polynomial of degree $(k-1)$. In short, the identity (A1) is true because the sum has the meaning of the $(n+1)$-st finite-difference derivative of an $n$-order polynomial.

For a more explicit solution, consider a polynomial

$$
f\left(x_{0}, x_{1}, \ldots, x_{n}\right)
$$

with $n+1$ variables. Suppose that the polynomial degree does not exceed $n$. Take the $(n+1)$-st finite difference derivative of the polynomial in the directions of $x_{0}, x_{1}, \ldots$, and $x_{n}$ that is a finite difference analogue of an ordinary derivative $\frac{\partial^{n+1} f\left(x_{0}, \ldots x_{n}\right)}{\partial x_{0} \partial x_{1} \ldots \partial x_{n}}$. The derivative has the form of the sum, over $2^{n+1}$ sequences $\left\{a_{0}, a_{1}, \ldots, a_{n}\right\}$ of zeroes and ones, of the values

$$
f\left(x_{0}+a_{0}, x_{1}+a_{1}, \ldots, x_{n}+a_{n}\right)
$$

taken with certain signs, $+/-$. The exact rule of signs is $(-1)^{a_{0}+a_{1}+\ldots+a_{n}}$ that is the sign is + if an odd number of the $a_{i}$ are ones, and - if even. In (A2), we assume that the finite difference $h$ is the same for any direction and $h=1$.

Thus we have:

$$
\sum_{\left\{a_{0}, a_{1}, \ldots, a_{n}=0,1\right\}}(-1)^{a_{0}+a_{1}+\ldots+a_{n}} f\left(x_{0}+a_{0}, x_{1}+a_{1}, \ldots, x_{n}+a_{n}\right) \equiv 0
$$

The statement is that if $f\left(x_{0}, x_{1}, \ldots, x_{n}\right)$ is a polynomial with a degree, not exceeding $n$, then the sum (A4) is equal to zero identically (i.e., for all values of $x_{0}, x_{1}, \ldots, x_{n}$ ). The proof is straightforward since a finite-difference partial derivative of a polynomial of degree $k$ is a polynomial of degree $(k-1)$ (just as it is for ordinary derivatives). Checking this for monomials essentially reduces to the one-variable fact that $(x+1)^{k}-x^{k}$ has degree of $(k-1)$. 
The identity (A1) is obtained by applying this formula to the polynomial

$$
f\left(x_{0}, x_{1}, \ldots, x_{n}\right)=\left(x_{0}+2 x_{1}+2^{2} x_{2}+\ldots+2^{n} x_{n}\right)^{n}
$$

whose $(n+1)$-st finite difference partial derivative is computed at the point

$$
\left(x_{0}, x_{1}, \ldots, x_{n}\right)=(1,0, \ldots, 0)
$$

To clarify the last statement note first that when $\left\{a_{0}, a_{1}, \ldots, a_{n}\right\}$ runs over all binary sequences, the number

$$
s=1+a_{0}+2 a_{1}+\ldots+2^{n} a_{n}
$$

runs over all integers from 1 to $2^{(n+1)}$. Second, note that for the function (A5) at a certain $s$, the expression $f\left(x_{0}+a_{0}, x_{1}+a_{1}, \ldots, x_{n}+a_{n}\right)$ in (A4), taken at the point (A6), reduces to the $s^{n}$ polynomial term. The elements of the sequence $\left\{r_{s}\right\}$ in (A1) can be obtained as factors $(-1)^{a_{0}+a_{1}+\ldots+a_{n}}$ in (A5). Then the rule of signs for $r_{s}$ is $r_{s}=+1$, when the binary code for $s$ has an even number of ones, and $r_{s}=-1$, when the number of ones in the binary code of $s$ is odd.

Note also that if in the sum (A1) $s^{n}$ is replaced with $(x+s)^{n}$, the sum still will be equal to 0 , and this is true for any value of $x$, and not necessarily for an integer; that is, $x$ could be any real number. This proves the statement made in the introduction that the scanning pattern, optimal for the suppression of the $n$-th order polynomial term of a drift [obeying identity (A1)], is also optimal for all polynomial terms of the order of less than $n$.

\section{ACKNOWLEDGMENTS}

The author is grateful to Alexander Givental, Dmitry Budker, Ralf Geckeler, Gennady Lebedev, Wayne McKinney, Howard Padmore, and Max Zolotorev for useful discussions and to Amparo Rommeveaux, Frank Siewert, Muriel Thomasset, and all Round Robin collaborators for providing the S3 reference mirror and the data of the Round Robin measurements. The Advanced Light Source is supported by the Director, Office of Science, Office of Basic Energy Sciences, Material Science Division, of the U.S. Department of Energy under Contract No. DE-AC02-05CH11231 at Lawrence Berkeley National Laboratory.

\section{Disclaimer}


Certain commercial equipment, instruments, or materials are identified in this document. Such identification does not imply recommendation or endorsement by the US Department of Energy, LBNL, or ALS, nor does it imply that the products identified are necessarily the best available for the purpose.

* Electronic address: vvyashchuk@lbl.gov

1 D. K. C. MacDonald, Noise and Fluctuations: An Introduction, (Dover, Mineola, NY, 2006).

2 Philippe Refregier, Noise Theory and Application to Physics, (Springer, New York, 2003).

3 V. V. Yashchuk, W. R. McKinney, T. Warwick, T. Noll, F. Siewert, T. Zeschke, R.D.Geckeler, "Proposal for a Universal Test Mirror for Characterization of Slope Measuring Instruments," Proc. of SPIE 6704, 67040A/1-12 (2007).

4 G. E. Harrison, M. A. Player, and P. G. H. Sandars, "A multichannel phase-sensitive detection method using orthogonal square waveforms," J. Phys. E: Sci. Instrum. 4, 750-754 (1971).

5 G. E. Harrison, P. G. H. Sandars, and S. J. Wright, "Experimental limit on the proton electric dipole moment," Phys. Rev. Lett. 22, 1263-1265 (1969).

6 M. A. Player and P. G. H. Sandars, "An experiment to search for an electric dipole moment in the ${ }^{3} P_{2}$ metastable state of xenon," J. Phys. B: Atom. Molec. Phys. 3, 1620-1265 (1970).

7 S. Altarev, Yu. V. Borisov, N. V. Borovikova, A. I. Egorov, S. N. Ivanov, E. A. Kolomensky, M. S. Lasakov, V. M. Lobashev, V. A. Nazarenko, A. N. Pirozhkov, AP A. P. Serebrov, Yu. V. Sobolev, E. V. Shulgina, "Search for the neutron electric dipole moment," Phys. Atom. Nuc. 59(12), 1152-70 (1996).

8 C. A. Baker, D. D. Doyle, P. Geltenbort, K. Green, M. G. D. van der Grinten, P. G. Harris, P. Iaydjiev, S. N. Ivanov, D. J. R. May, J. M. Pendlebury, J. D. Richardson, D. Shiers, K. F. Smith, "Improved experimental limit on the electric dipole moment of the neutron," Phys. Rev. Lett. 97(14), 131801/1-4 (2006).

9 B. C. Regan, E. D. Commins, C. J. Schmidt, and D. DeMille, "New Limit on the Electron Electric Dipole Moment,” Phys. Rev. Lett. 88(12), 071805/1-4 (2002).

10 W. C. Griffith, M. D. Swallows, T. H. Loftus, M. V. Romalis, B. R. Heckel, and E. N. Fortson, "Improved Limit on the Permanent Electric Dipole Moment of 199Hg," Phys. Rev. Lett. 102(4), 101601/1-4 (2009). 
11 S. A. Murthy, D. Krause, Z. L. Li, and L. R. Hunter, "New limits on the electron electric dipole moment from cesium," Phys. Rev. Lett. 63, 965-968 (1989).

12 Edward A. Hinds and P. G. Sandars, "Experiment to search for P- and T-violating interactions in the hyperfine structure of thallium fluoride," Phys. Rev. A 21, 480-483 (1980).

13 J. J. Hudson, B. E. Sauer, M. R. Tarbutt, and E. A. Hinds, "Measurement of the Electron Electric Dipole Moment Using YbF Molecules,” Phys. Rev. Lett. 89, 023003/1-4 (2002).

14 B. J. Heidenreich, O. T. Elliott, N. D. Charney, K. A. Virgien, A. W. Bridges, M. A. McKeon, S. K. Peck, D. Krause, J. E. Gordon, L. R. Hunter, and S. K. Lamoreaux, "Limit on the Electron Electric Dipole Moment in Gadolinium-Iron Garnet," Phys. Rev. Lett. 95, 253004/1-4 (2005).

15 V. V. Yashchuk, J. L. Kirschman, G. Y. Morrison, B. V. Smith, E. E. Domning, F. Siewert, T. Zeschke, R. D. Geckeler, A. Just, "Sub-microradian Surface Slope Metrology with the ALS Developmental Long Trace Profiler," (to be published).

16 F. Siewert, T. Noll, T. Schlegel, T. Zeschke, and H. Lammert, "The Nanometer Optical Component Measuring machine: a new Sub-nm Topography Measuring Device for X-ray Optics at BESSY," Proc. of AIP 705, 847-850 (2004).

17 A. Pramanik, Electro-Magnetism: Theory and Applications (Prentice-Hal India, 2004).

18 A. P. J. van Deursen, "Visualization of induced electric fields," Am. J. Phys. 73(12), 1099-1106 (2005).

19 P. Takacs, S. N. Qian and J. Colbert, Design of a long trace surface profiler, Proc. of SPIE 749, 59-64 (1987).

20 S. Qian, W. Jark, P. Z, Takacs, "The Penta prism LTP: a Long Trace Profiler with stationary optical head and moving Penta-prism," Rev. Sci. Instrum. 66(3), 2562-2569 (1995).

21 R. D. Geckeler, "ESAD Shearing Deflectometry: Potentials for Synchrotron Beamline Metrology," Proc. of SPIE 6317 - 63171H/1-12 (2006).

22 W. R. McKinney, S. C. Irick, and D. L. J. Lunt, Optics for High-brightness Synchrotron Radiation Beamlines, Proc. of SPIE 1740, 154-160 (1992).

23 A high quality 1280-m spherically shaped reference mirror, labeled S3, belongs to the SOLEIL synchrotron (Muriel Thomasset), and is used for intensive Round Robin cross-comparisons of slope measuring profilers around the world. ${ }^{24,25}$

24 Rommeveaux A, Thomasset M, Cocco D, Siewert F., First report on a European round robin for slope measuring profilers, Proc. of SPIE 5921 - 592101 (2005). 
25 F. Siewert, L. Assoufid, D. Cocco, O. Hignette, S. Irick, H. Lammert, W. McKinney, H. Ohashi, F. Polack, S. Qian, S. Rah, A. Rommeveaux, V. Schnherr, G. Sostero, P. Takacs, M. Thomasset, K. Yamauchi, V. Yashchuk, T. Zeschke, Global high-accuracy inter-comparison of slope measuring instruments, AIP Conference on Synchrotron Radiation Instrumentation SRI-2006, III Workshop on Optical Metrology, CD Proceedings (Daegu, South Korea, May 27 - June 03, 2006).

26 The S3 mirror specification data and the result of Round Robin measurements with the mirror are courtesy of Amparo Rommeveaux and Frank Siewert.

27 The idea of the proof of the identity presented in the Appendix was suggested by Alexander Givental. 


\section{FIGURES}

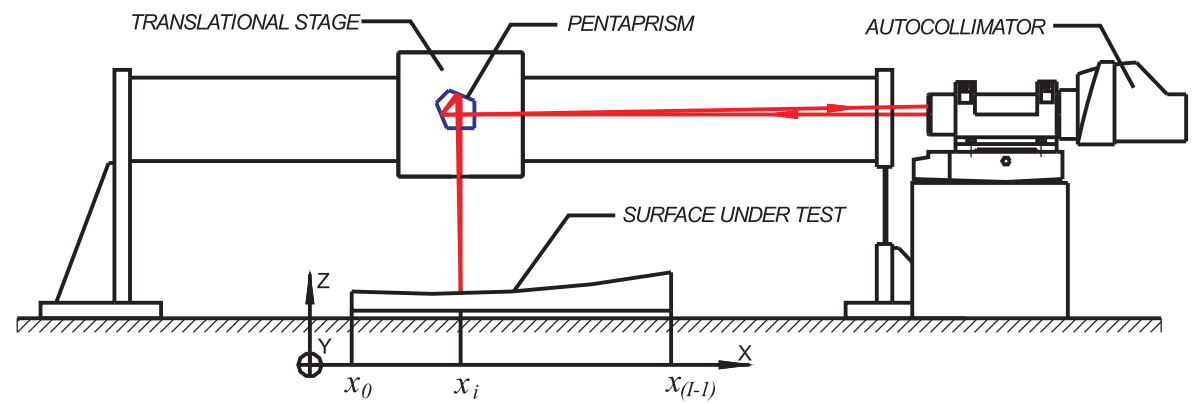

FIG. 1: Simplified schematic of slope measurements with the ALS DLTP instrument. The optical slope sensor of the DLTP is a high-precision autocollimator. In the course of a measurement, scanning along a SUT is performed by translating a pentaprism in the forward (increase of the $\mathrm{x}$-coordinate) or backward direction. 

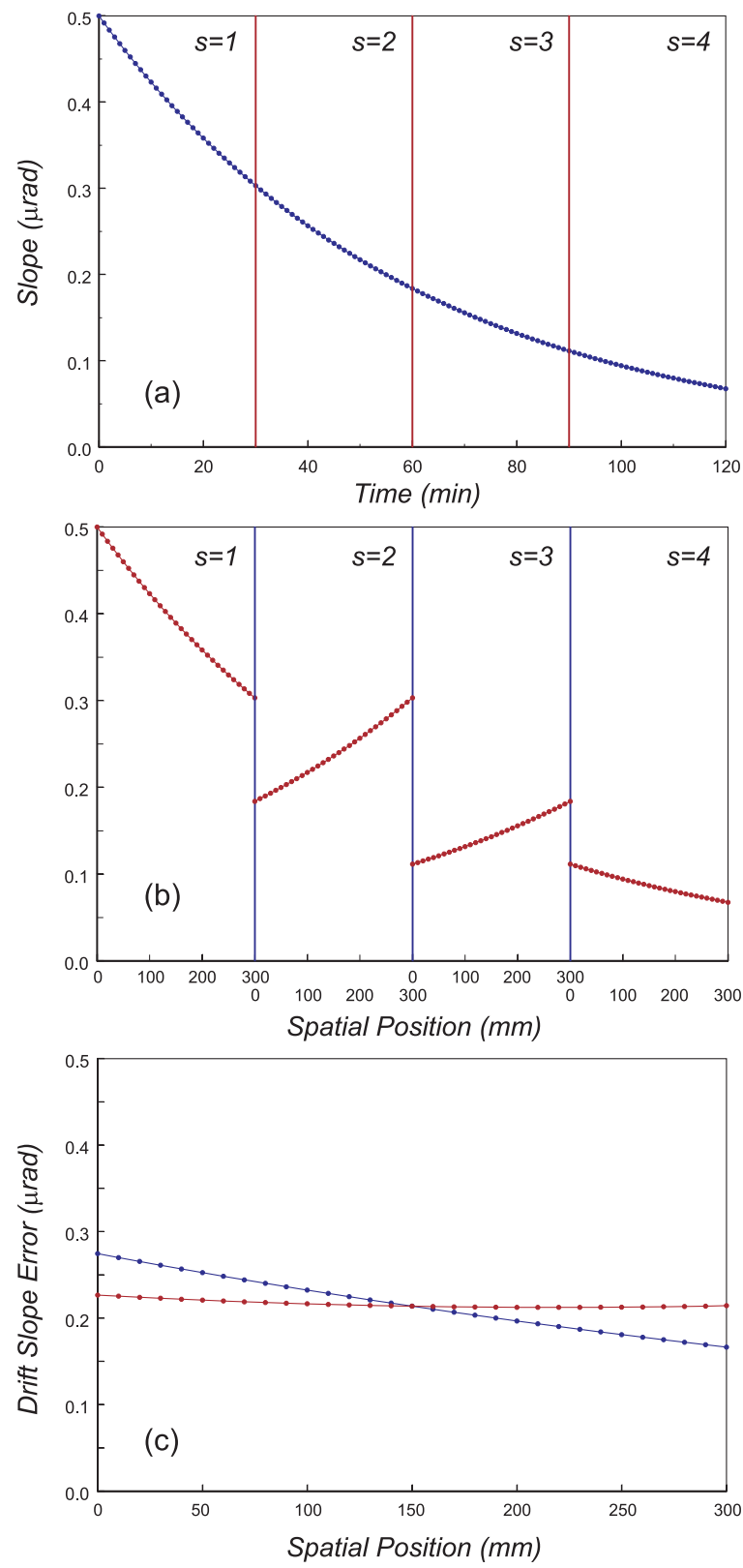

FIG. 2: Suppression of the exponential drift described by Eq. (41) with the parameters $D_{0}=0.5$ $\mu \mathrm{rad}$ and $\tau=60 \mathrm{~min}$. (a) Time dependence of the exponential drift. (b) The same drift is presented as a dependence on the spatial position along a SUT when four scans are performed according to the scan strategy $\left\{r_{s}^{+}\right\}=\{+1,-1,-1,+1\} \equiv\{F, B, B, F\}$. (c) The resulting traces of the slope error due to the exponential drift measured in four succesive scans performed in one direction (blue line and points) and in four scans performed according to the optimal scanning strategy (red line and points). 


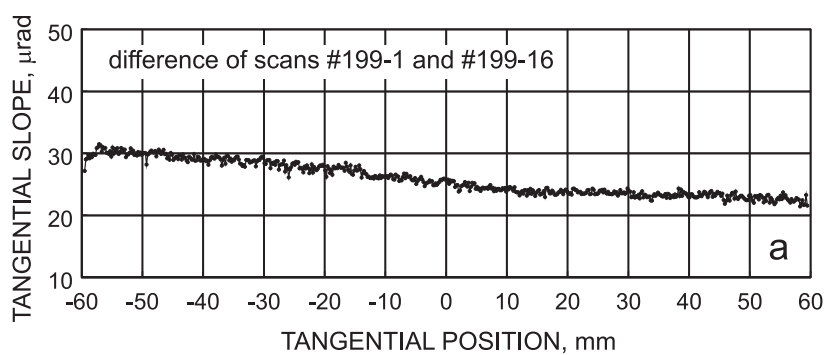

FIG. 3: DLTP set-up drift as a difference of the slope traces measured in the first (1st) and the last (16th) scans of the measured series \#199 (see text). The number of the series, \#199, relates to a current number of the measurement series when it was performed with the DLTP.

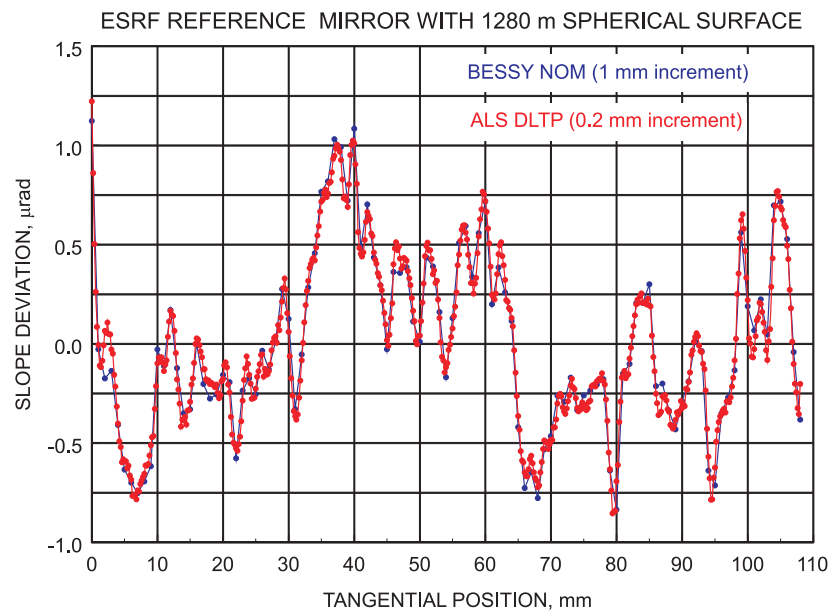

FIG. 4: The red points and line are DLTP measurement with the S3 reference mirror. The blue points and line presents the residual slope trace obtained with BESSY NOM (courtesy of Frank Siewert). 